The role of explicit solvent molecules in the calculation of NMR chemical shifts of glycine in water

Caputo, María Cristina; Provasi, Patricio F.; Sauer, Stephan P. A.

Published in:

Theoretical Chemistry Accounts

DOI:

10.1007/s00214-018-2261-9

Publication date:

2018

Document version

Peer reviewed version

Citation for published version (APA):

Caputo, M. C., Provasi, P. F., \& Sauer, S. P. A. (2018). The role of explicit solvent molecules in the calculation of NMR chemical shifts of glycine in water. Theoretical Chemistry Accounts, 137(7), [88].

https://doi.org/10.1007/s00214-018-2261-9 


\title{
The role of explicit solvent molecules in the calculation of NMR chemical shifts of glycine in water
}

\author{
María C. Caputo - Patricio F. Provasi · \\ Stephan P.A. Sauer
}

Received: date / Accepted: date

\begin{abstract}
We present the results of a computational study of the NMR properties of glycine in water solution at the level of density functional theory employing the B3LYP functional and the 6-31G(d,p) and pcSseg-2 basis sets, describing the solvent either via the PCM continuous solvation model or PCM with additional explicit water molecules hydrogen bonded to the solute. We observe that the solvent causes considerable changes in the predicted magnetic shieldings and that the results depend significantly on the number of solvent molecules included in the quantum mechanical treatment.
\end{abstract}

Keywords Chemical shift $\cdot$ PCM $\cdot$ pcSseg-2 basis sets

\section{Introduction}

Amino acids are some of the fundamental bricks of life carrying out much of the functions in cells and therefore in living organisms. Glycine, in particular,

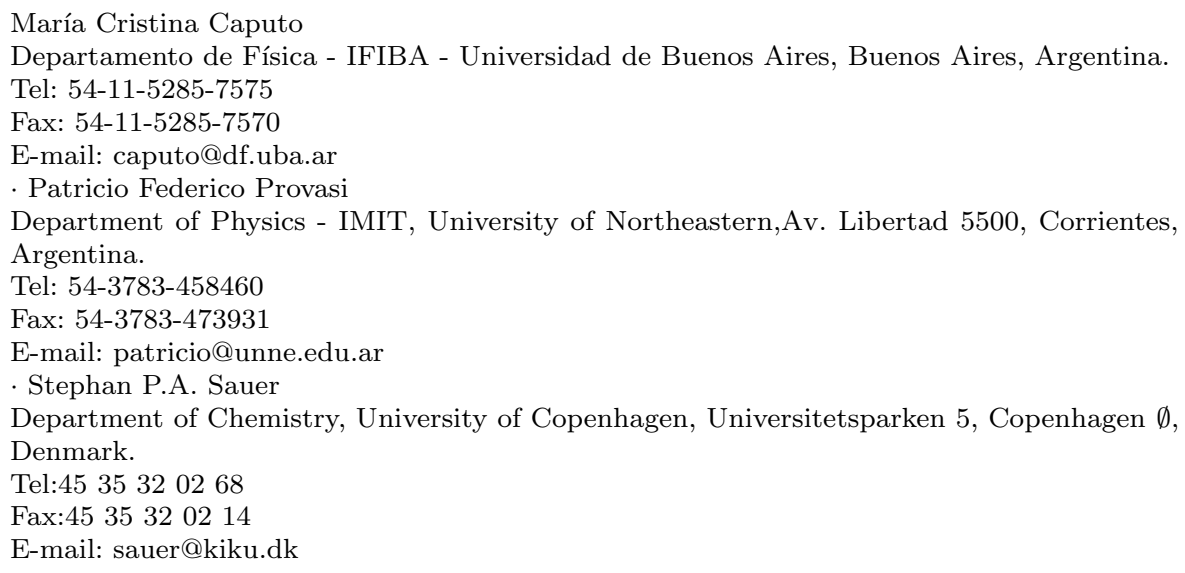


is the smallest amino acid allowing a great diversity of theoretical studies, [1-14] which is stimulated by the fact that some of its properties are known experimentally. Recently the investigation on the micro complexes of water and small amino acids has gained a renewed interest in particular, as even when they are measured in gas phase there is a micro solvation of the molecule of interest. $[15,16]$

Among the many studies devoted to glycine some were aimed at elucidating the mechanisms of tautomerization where the proton transfer of the neutral glycine, which is stable in the gas phase, to the zwitterionic conformer, which is stable in aqueous solution, has an estimated free energy barrier of about $7.3 \frac{\mathrm{kcal}}{\mathrm{mol}}[1-3,5,6,11,17-21]$. Other recent work was aimed at studying small clusters of Gly. $\left(\mathrm{H}_{2} \mathrm{O}\right)_{n}$ complexes in order to elucidate structures and binding energies $[22,23]$.

The three most stable conformers of neutral glycine in gas phase were determined by Császár [24], Godfrey et al [25], Sauer et al [7], and Aikens and Gordon [26]. In this work we found the same three structures in the same energetic order as in the previous studies with the exception of Godfreyet $a l$ [25]. The second most stable neutral conformation $(\mathrm{N})$ is depicted in Figure 1 (left), where the $\mathrm{C}-\mathrm{N}$ and $\mathrm{O}-\mathrm{H}$ bonds are antiperiplanar to the $\mathrm{C}=\mathrm{O}$ bond. $\mathrm{Of}$ the three most stable neutral conformers only this structure is converted to the zwitterion (Z), shown in Figure 1 (right). The latter can be obtained from the corresponding $\mathrm{N}$ conformer by geometry optimization using the Polarizable Continuum Model (PCM). These two structures are respectively the most stable geometries we found for $\mathrm{N}$ - and Z-glycine when including a continuum polarizable medium.
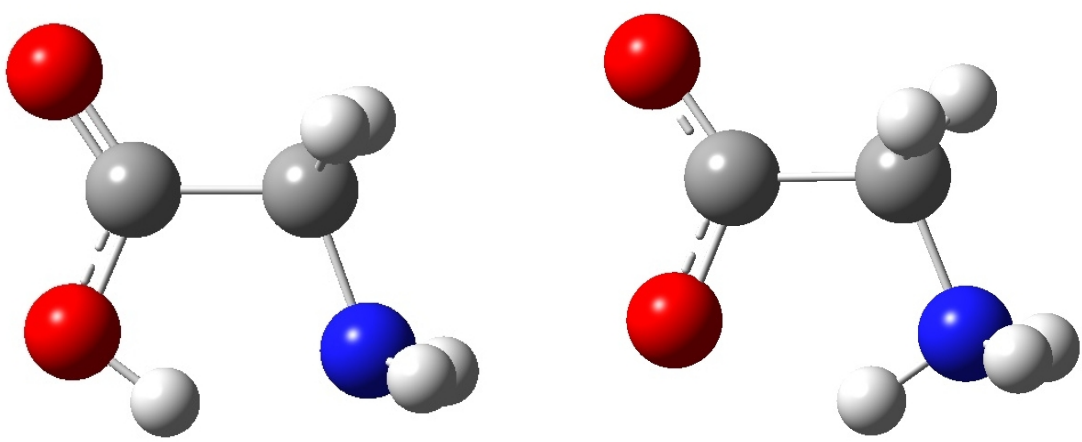

Fig. 1 Optimized geometries of neutral (left) and zwitterionic (right) glycine forms using B3LYP/6-31G(d,p)

The purpose of this work is to investigate the NMR properties of glycine in water solution, studying in detail the applicability of the PCM continuous solvation model approximation [27-34], as compared to the introduction of explicit hydrogen-bonded (HB) solvent molecules in calculations of NMR nuclear shieldings . 


\section{Computational details}

All the calculations were carried out with Gaussian09 program [35], using density functional theory (DFT) with the B3LYP exchange correlation [36, 37], in a liquid solvent described by the PCM approximation [38].

Calculations of the structures and energies of the complexes were carried out with the $6-31 \mathrm{G}(\mathrm{d}, \mathrm{p})$ basis set, with 120 and 25 basis functions for the glycine and water molecules, respectively $[39,40]$. The geometry optimizations were started from several conformers and explicit solvent molecule positions. Each additional water molecule was placed so that it could form at least one hydrogen bond to a vacant hydrophilic site in glycine. For each case, about 20 different starting positions consistent with the HB criterion in Ref. [6] were tried in order to reduce the risk of ending in a local minimum during the optimization procedure. In practice, most of the starting configurations converged nevertheless to the same single geometry.

Calculations of the nuclear magnetic shieldings were performed using Gaussian09 both at the same level of the theory as mentioned above and with a specialized basis set, namely pcSseg-2 for shieldings [41].

The theory of nuclear shielding constants $(\sigma)$ [42] and different computational methods for calculating them have been described extensively in the literature [43-46]. In the present work they were calculated employing the gauge including atomic orbitals (GIAOs) method [47] due to its reduced basis set dependence as compared to e.g. the CTOCD-DZ method [48,49].

The magnetic properties depend on the number of explicit water molecules taken into account, which we vary from zero to a full first solvation layer. Considering only $\mathrm{H}_{2} \mathrm{O}$ molecules directly hydrogen-bonded to the solute, the number of solvent molecules necessary to reach saturation of the two hydrophilic groups of glycine (the amino and the acidic ends) vary a bit according to the conditions established for the hydrogen bond formation. Following Campo [6], we require a maximum distance of $2 \AA$ between donor and acceptor of the glycine and water molecules for a hydrogen bond, and a minimum angle of $145^{\circ}$ formed by the atoms $\mathrm{X}_{\mathrm{donor}}-\mathrm{H}-\mathrm{Y}_{\text {acceptor }}$, where $\mathrm{X}$ and $\mathrm{Y}$ are either both oxygen atoms, or an oxygen and a nitrogen atom.

\section{Results}

The NMR properties for glycine in water solution can be calculated by accounting for solvent effects on average using the PCM solvation model. An approach to study the consistency of these predictions consists in comparing the results to those obtained by incorporating explicit water molecules hydrogen-bonded to glycine and embedding the ensuing complex in the continuous, homogeneous and polarizable medium of PCM. The positions where water molecules can be hydrogen-bonded to glycine are found by searching for stable local energy minima structures, that pass the frequency test. 
3.1 Structures of hydrogen-bonded complexes

Basically four hydrophilic sites were identified in N-glycine: the trans and cis oxygen atoms, i.e. anti- or syn-periplanar to the $\mathrm{C}-\mathrm{N}$ bond, (henceforth respectively denoted as $\mathrm{OT}$ and $\mathrm{OC}$ ), the hydrogen atoms in the amino group (HN) and a water molecule simultaneously hydrogen-bonded to the carboxylic hydrogen and the nitrogen atom in the amino group (AA):
(1) OT, trans-oxygen:
$\mathrm{H}-\mathrm{O}-\mathrm{H} \cdots \mathrm{O}=\mathrm{C}$
(2) OC, cis-oxygen:
$\mathrm{H}-\mathrm{O}-\mathrm{H} \cdots(\mathrm{OH})-\mathrm{C}=\mathrm{O}$
(3) $\mathrm{HN}$, amino group hydrogen:
$\mathrm{H}_{2}-\mathrm{O} \cdots \mathrm{H}-\mathrm{N}$
(4) AA, acid and amino groups: $\mathrm{C}-\mathrm{O}-\mathrm{H} \cdots(\mathrm{OH})-\mathrm{H} \cdots \mathrm{N}$

Although the last case actually comprises two hydrophilic positions, all attempts to find a local energy minimum with a solvent molecule bonded to only one of these positions resulted in a $\mathrm{H}_{2} \mathrm{O}$ molecule hydrogen-bonded to both sites. The positions of the four hydrophilic sites are depicted in Figure 2.
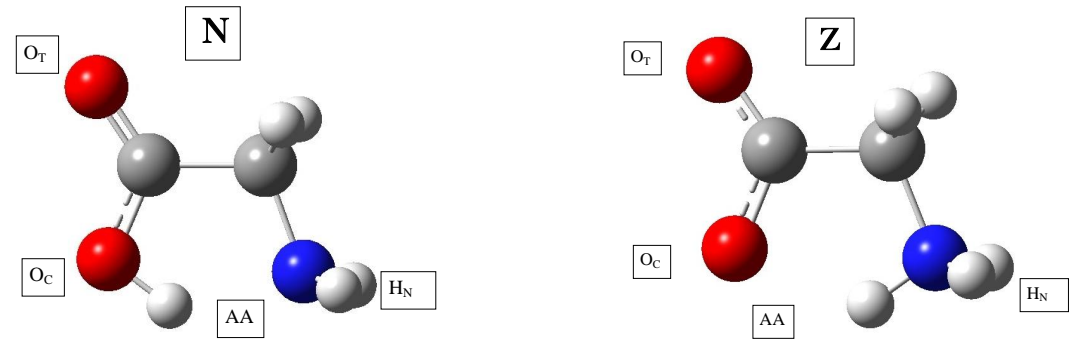

Fig. 2 Diagram of the possible H-bond sites between glycine-N/glycine-Z in water

In the case of the zwitterionic conformer the situation is similar, the only differences being that the cis-oxygen (OC) is no longer protonated, and that the doubly hydrogen-bonded case (AA) now corresponds to simultaneous bonds to an aminonium hydrogen atom and to the cis-oxygen.

Glycine, in either its N- or Z- conformer, has five magnetically distinct positions occupied by non-hydrogen nuclei: the cis and trans oxygen atoms, the carboxylic and methylenic carbon atoms, and the nitrogen atom. In the following these are respectively denoted as $\mathrm{O}_{c}, \mathrm{O}_{t}, \mathrm{C}_{c}, \mathrm{C}_{m}$, and $\mathrm{N}$.

3.2 Nuclear magnetic shieldings for the non-hydrogen nuclei in glycine in water solution

The nuclear magnetic shieldings for the non-hydrogen nuclei in glycine in water solution calculated at the DFT/B3LYP level within the PCM continuous solvation model and the pcSseg- 2 basis set are summarized in Table 1 for the 
$\mathrm{N}$ - and Z-conformers. The table also shows, for future reference, the magnetic shieldings calculated with the smaller 6-31G(d,p) basis set. As expected for absolute shieldings, the results differ substantially between the two cases. However, the relevant property, i.e. the chemical shift between the two conformers exhibits a much small basis set dependence of 1-4 ppm apart from $\mathrm{O}_{c}$ with $11 \mathrm{ppm}$.

Table 1 Absolute nuclear magnetic shieldings (in ppm) for the non-hydrogen nuclei in glycine in water solution calculated at the DFT/B3LYP level using PCM with the pcSseg-2 and $6-31 G(d, p)$ basis sets

\begin{tabular}{cccccrr}
\hline Conformer & Basis Set & $\mathrm{C}_{c}$ & $\mathrm{C}_{m}$ & $\mathrm{~N}$ & \multicolumn{1}{c}{$\mathrm{O}_{c}$} & \multicolumn{1}{c}{$\mathrm{O}_{t}$} \\
\hline \multirow{3}{*}{ N-Glycine } & 6-31G(d,p) & 15.0 & 147.9 & 240.5 & 128.9 & 13.2 \\
& pcSseg-2 & -17.2 & 131.8 & 226.3 & 97.5 & -34.3 \\
\hline \multirow{2}{*}{ Z-Glycine } & 6-31G(d,p) & 23.0 & 146.6 & 226.6 & 45.2 & 52.5 \\
& pcSseg-2 & -8.5 & 130.8 & 209.0 & 2.9 & 9.0 \\
\hline
\end{tabular}

The reliability of the PCM approach to describe the surrounding solvent medium can be tested by incorporating water molecules until saturation of all possible hydrophilic sites. It is interesting to see how this process evolves while adding solvent molecules one-by-one, i.e. to see at what point an asymptotic regime is reached. Our procedure was sequential. First, we found that for N-glycine the most stable Gly. $\left(\mathrm{H}_{2} \mathrm{O}\right)$ complex calculated with PCM corresponds to a $\mathrm{H}_{2} \mathrm{O}$ molecule occupying the OT position. Next we looked for the Gly. $\left(\mathrm{H}_{2} \mathrm{O}\right)_{2}$ complex with lowest energy having at least one water molecule at the OT position. In this way, the procedure was continued, where the most stable Gly. $\left(\mathrm{H}_{2} \mathrm{O}\right)_{n}$ structure was obtained while respecting the configuration of the previously found Gly. $\left(\mathrm{H}_{2} \mathrm{O}\right)_{n-1}$ complex. We found that a total of $n=8$ water molecules can be added having each at least one $\mathrm{HB}$ to $\mathrm{N}$-glycine. The result of sequentially occupied hydrophilic positions is OT, OC, AA, OC, HN, OT, HN, OT. This means that, upon saturation, the number of water molecules hydrogen-bonded to the OT, OC, HN and AA hydrophilic sites of N-Glycine are 3,2, 2 and 1, respectively. The geometries of these complexes can be found in the supplementary material.

Tables 2 and 3 present the cumulative changes in the predicted shieldings in $\mathrm{N}$ - and Z-glycine with respect to the bare PCM calculation, $\Delta \sigma_{n}=$ $\sigma\left[\right.$ Gly. $\left.\left(\mathrm{H}_{2} \mathrm{O}\right)_{n}\right]-\sigma[\mathrm{Gly}]$, as explicit solvent molecules hydrogen-bonded to glycine in a PCM environment are included in the quantum mechanical treatment. Our calculations include as limiting values the complexes Gly. $\left(\mathrm{H}_{2} \mathrm{O}\right)_{9}$ and Gly. $\left(\mathrm{H}_{2} \mathrm{O}\right)_{10}$, where the water molecules beyond $n=8$ are solely $\mathrm{H}$-bonded to solvent molecules. The results are presented graphically in the left panels of Figures 3, 4 and 5 for the $\mathrm{N}_{-} \mathrm{C}_{c}-\mathrm{C}_{m}, \mathrm{O}_{c}$ and $\mathrm{O}_{t}$ nuclei for $\mathrm{N}$-glycine and in the right panels for Z-glycine. 
Table 2 The magnetic shielding (in ppm) for the non-hydrogen nuclei in N-glycine in water solution: difference between the results using $n$ and zero explicit solvent molecules both in a PCM environment calculated using the pcSseg-2 basis set.

\begin{tabular}{ccrrrrr}
\hline Hydrophilic site & $n\left(\mathrm{H}_{2} \mathrm{O}\right)$ & \multicolumn{1}{c}{$\mathrm{C}_{c}$} & $\mathrm{C}_{m}$ & \multicolumn{1}{c}{$\mathrm{N}$} & \multicolumn{1}{c}{$\mathrm{O}_{c}$} & \multicolumn{1}{c}{$\mathrm{O}_{t}$} \\
\hline $\mathrm{OT}$ & 1 & -1.5 & 0.1 & 0.1 & -3.4 & 5.7 \\
$\mathrm{OC}$ & 2 & 0.2 & 0.0 & -0.2 & -4.2 & 1.3 \\
$\mathrm{AA}$ & 3 & 1.0 & -5.0 & -5.5 & -12.7 & -27.9 \\
$\mathrm{OC}$ & 4 & 1.6 & -5.1 & -5.6 & -14.6 & -38.7 \\
$\mathrm{HN}$ & 5 & 1.1 & -4.5 & -7.5 & -15.0 & -36.4 \\
$\mathrm{OT}$ & 6 & -0.8 & -4.1 & -7.7 & -19.4 & -28.9 \\
$\mathrm{HN}$ & 7 & -0.8 & -3.8 & -9.3 & -18.2 & -32.4 \\
$\mathrm{OT}$ & 8 & -3.0 & -4.1 & -9.1 & -24.5 & -27.1 \\
Ext & 9 & -3.1 & -4.0 & -9.4 & -23.8 & -24.2 \\
Ext & 10 & -2.6 & -3.9 & -10.3 & -21.3 & -30.4 \\
\hline
\end{tabular}

Table 3 The magnetic shielding (in ppm) for the non-hydrogen nuclei in Z-glycine in water solution: difference between the results using $n$ and zero explicit solvent molecules both in a PCM environment calculated using the pcSseg-2 basis set.

\begin{tabular}{ccccccc}
\hline Hydrophilic site & $n\left(\mathrm{H}_{2} \mathrm{O}\right)$ & $\mathrm{C}_{c}$ & $\mathrm{C}_{m}$ & $\mathrm{~N}$ & $\mathrm{O}_{c}$ & $\mathrm{O}_{t}$ \\
\hline $\mathrm{AA}$ & 1 & -1.5 & -0.7 & -0.6 & -0.5 & -11.7 \\
$\mathrm{OT}$ & 2 & -1.7 & -0.5 & -0.1 & -5.6 & -10.3 \\
$\mathrm{OC}$ & 3 & -1.8 & -0.5 & -0.2 & -7.9 & -10.1 \\
$\mathrm{HN}$ & 4 & -2.2 & 0.3 & -1.4 & -4.2 & -15.7 \\
$\mathrm{OC}$ & 5 & -2.9 & 0.6 & -2.7 & -1.7 & -23.0 \\
$\mathrm{OT}$ & 6 & -3.8 & 0.3 & -1.6 & -3.6 & -27.1 \\
$\mathrm{HN}$ & 7 & -4.4 & 1.3 & -3.5 & -3.5 & -24.5 \\
$\mathrm{OT}$ & 8 & -5.4 & 1.1 & -3.4 & -13.7 & -20.9 \\
Ext & 9 & -5.8 & 1.1 & -3.4 & -14.3 & -20.0 \\
Ext & 10 & -5.7 & 1.0 & -3.3 & -12.3 & -21.1 \\
\hline
\end{tabular}

Figures 4 and 5, in addition to the analysis with the pcSseg-2 basis set (solid line), include the values calculated with the smaller 6-31G(d,p) basis set (dashed line). It is satisfying to see that the results for $\Delta \sigma_{n}$ are very similar for both basis sets, indicating that the larger set is not essential for evaluating the validity of PCM as a description of the solvent.

The pattern that emerges as water molecules are added to the four hydrophilic positions is that the predicted shieldings in the end always decrease or in the single case of $\mathrm{C}_{m}$ in Z-glycine stay basically constant. Furthermore, the shieldings tend to converge with increasing number of explicitly considered solvent molecules, maybe with the exception of $\mathrm{O}_{t}$ in the $\mathrm{N}$-conformer. In both conformers the largest effect is observed for $\mathrm{O}_{t}$ and $\mathrm{O}_{c}$. The trans-oxygen is 

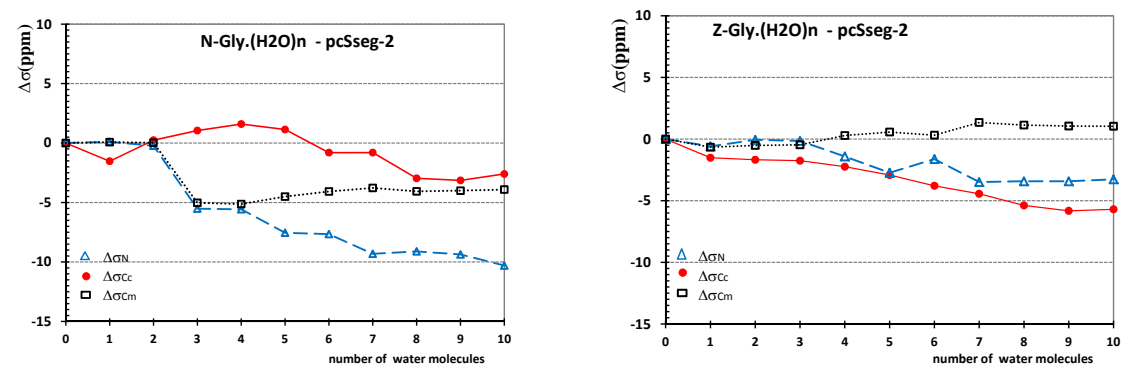

Fig. 3 Change in the magnetic shieldings (in ppm) of the carbon and nitrogen nuclei for N(left) and Z- (right) glycine in water solution, as explicit solvent molecules are incorporated in the calculation, using the pcSseg-2 basis set.
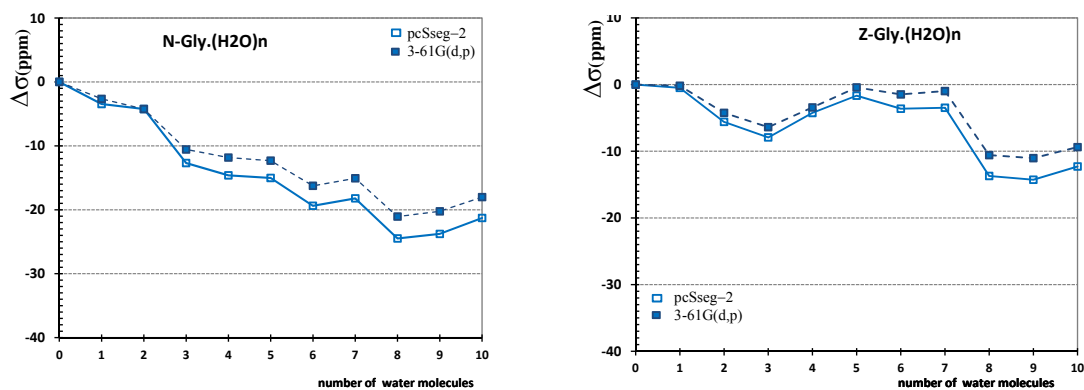

Fig. 4 Change in the magnetic shieldings (in ppm) of cis-oxygen for N- (left) and Z- (right) glycine in water solution, as explicit solvent molecules are incorporated in the calculation, using the pcSseg-2 (solid line) and the 3-61G(d,p) (dashed line) basis sets.
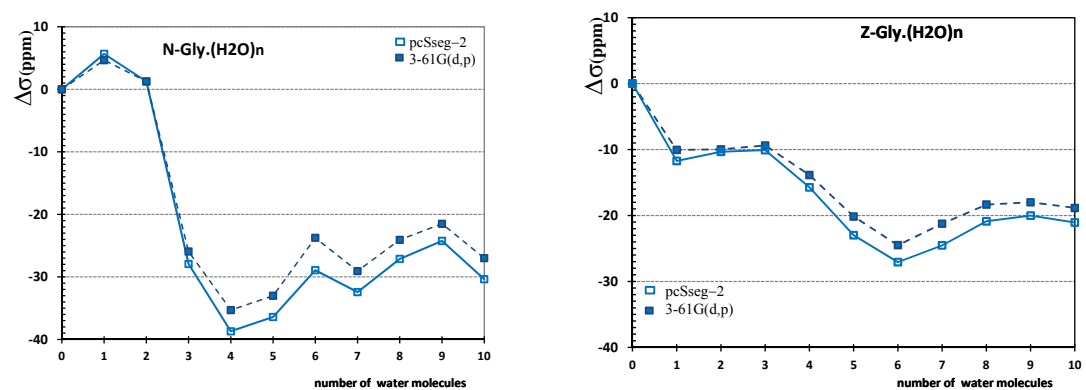

Fig. 5 Change in the magnetic shieldings (in ppm) of trans-oxygen for N- (left) and Z(right) glycine in water solution, as explicit solvent molecules are incorporated in the calculation, using the pcSseg-2 (solid line) and the $3-61 \mathrm{G}(\mathrm{d}, \mathrm{p})$ (dashed line) basis sets.

the most sensitive to the presence of explicit water molecules and thus the nucleus, where the disagreement with the bare PCM prediction is the largest.

For the $\mathrm{N}$-conformer the $\mathrm{O}_{t}$ nucleus is systematically shielded (deshielded) when adding solvent molecules at position OT (OC), with an average change of $+6 \mathrm{ppm}(-6 \mathrm{ppm})$. The positive value is the expected behavior for an electronegative atom when hydrogen-bonded to hydrogen. Addition of $\mathrm{H}_{2} \mathrm{O}$ to 
the AA position brings about a large deshielding effect $(\Delta \sigma=-26 \mathrm{ppm})$, a result that we find surprising. The cis-oxygen is mainly affected by explicit water molecules at the AA and OT sites, with deshieldings of $\Delta \sigma=-7$ and $\Delta \sigma \simeq-4$ respectively, for a global change of $\Delta \sigma=-20 \mathrm{ppm}$. The decrease in shielding is smaller but also significant for nitrogen $(\Delta \sigma=-8 \mathrm{ppm})$, driven by the inclusion of explicit water molecules at the AA $(\Delta \sigma=-4 \mathrm{ppm})$ and $\mathrm{HN}(\Delta \sigma=-2 \mathrm{ppm})$ positions. The effect on the carbon nuclei is smaller, $\Delta \sigma=-3 \mathrm{ppm}$ for $\mathrm{C}_{m}$ (only $\mathrm{H}_{2} \mathrm{O}$ at $\mathrm{AA}$ is relevant) and $\Delta \sigma=-2 \mathrm{ppm}$ for $\mathrm{C}_{c}$ because of cancelations between the contributions of water at the OT and OC sites.

For the Z-conformer, the distribution of solvent molecules at the hydrophilic sites is the same, but the order in which they are occupied differs, as detailed in Table 3. The two main effects are a reduction in shielding of $-19 \mathrm{ppm}$ and $-10 \mathrm{ppm}$ for the trans and the cis-oxygen nuclei, respectively. The total result for $\mathrm{O}_{t}$ arises from three negative contributions, $\mathrm{AA}(\Delta \sigma \simeq-10)$, $\mathrm{OC}(\Delta \sigma \simeq$ $-5 \mathrm{ppm})$ and $\mathrm{HN}(\Delta \sigma \simeq-1 \mathrm{ppm})$, and one positive, OT $(\Delta \sigma \simeq+2.5 \mathrm{ppm})$. The global result for $\mathrm{O}_{c}$ is smaller because of comparable positive and negative contributions, $\mathrm{OT}(\Delta \sigma \simeq-6.5 \mathrm{ppm})$ and $\mathrm{HN}(\Delta \sigma \simeq+4 \mathrm{ppm})$. For the carboxyl carbon the total change is $\Delta \sigma \simeq-5 \mathrm{ppm}$ with comparable contributions from water at each site, for the methylenic carbon the effect is negligible, and for nitrogen it is $\Delta \sigma \simeq-1 \mathrm{ppm}$ where the only contribution is from the $\mathrm{HN}$ site.

Summarizing we find that in particular for the oxygen nuclei, but also for nitrogen the converged results with explicit hydrogen-bonded water molecules differ substantially from the PCM only results, meaning that the continuous polarizable medium approximation is not adequate for predicting NMR shieldings of a solution of glycine in water. The effect is thought larger for the neutral conformer than for the zwitterionic structure.

3.3 Influence of water molecules at each hydrophilic site on the nuclear magnetic shieldings

The results in Tables 2 and 3 report the change in the predicted magnetic shieldings when adding to the quantum region a water molecule hydrogenbonded to the hydrophilic sites in a particular Gly. $\left(\mathrm{H}_{2} \mathrm{O}\right)_{n}$ complex. In this section we discuss how $\Delta \sigma(I, J)$, the change in the magnetic shielding at nucleus $I\left(I=\mathrm{O}_{c}, \mathrm{O}_{t}, \mathrm{C}_{c}, \mathrm{C}_{m}, \mathrm{~N}\right)$ when adding an explicit hydrogen-bonded solvent molecule at hydrophilic site $J(J=$ OC, OT, HN, AA), depends on the presence of other solvent molecules hydrogen-bonded to the solute.

We thus consider the changes in the shielding brought about by the addition of $\mathrm{H}_{2} \mathrm{O}$ to the same hydrophilic site in different Gly. $\left(\mathrm{H}_{2} \mathrm{O}\right)_{n}$ complexes, with $n=0,7$. Furthermore we have to consider that, for a given value of $n$, there are several possible distributions of the $n$ solvent molecules around glycine. For instance, we find 9 distinct Gly. $\left(\mathrm{H}_{2} \mathrm{O}\right)_{3}$ complexes to which a $\mathrm{H}_{2} \mathrm{O}$ molecule can be added at the $\mathrm{OT}$ site, yielding 9 different Gly. $\left(\mathrm{H}_{2} \mathrm{O}\right)_{4}$ 
complexes and thus nine $\Delta \sigma(I, \mathrm{OT})$ results for each nucleus $I$. Considering all values of $n$, this amounts to a total of 31 different complexes to which an additional solvent molecule can be added at the OT site.

In order to summarize the values of $\Delta \sigma(I, J)$, we have computed $(\Delta \sigma)_{i}$ for each possible starting structure, and evaluated their sample mean $\langle\Delta \sigma\rangle$ and standard deviation SD,

$$
\begin{aligned}
\langle\Delta \sigma\rangle & =\frac{\sum_{i=1}^{m}(\Delta \sigma)_{i}}{m} \\
\mathrm{SD} & =\sqrt{\frac{\sum_{i=1}^{m}\left[(\Delta \sigma)_{i}-\langle\Delta \sigma\rangle\right]^{2}}{m-1}}
\end{aligned}
$$

Here $m$ is the total number of different Gly. $\left(\mathrm{H}_{2} \mathrm{O}\right)_{n}$ complexes $(n=0,7)$ to which an additional solvent molecule can be added at the $J$ site. The values of $m$ are 28, 31, 30 and 25 respectively for the OC, OT, HN and AA hydrophilic sites.

Given the computational cost required to evaluate $\Delta \sigma$ for the large number of possible Gly. $\left(\mathrm{H}_{2} \mathrm{O}\right)_{n}$ complexes, and the fact that the predictions of $\Delta \sigma$ have been found to be similar for the pcSseg-2 and 6-31G(d,p) basis sets, we used the latter for the studies presented in this section.

Tables 4 and 5 summarizes the average change in the NMR shielding at each hon-hydrogen nucleus due to the addition of $\mathrm{H}_{2} \mathrm{O}$ molecules to the different hydrophilic sites for the $\mathrm{N}$ - and Z- conformers.

Table 4 The change in magnetic shielding (in ppm) for the non-hydrogen nuclei in N-glycine due to the addition of a $\mathrm{H}_{2} \mathrm{O}$ molecule at the solute hydrophilic sites (see description in the text)

\begin{tabular}{lrrrrr}
\hline Site & \multicolumn{1}{c}{$\mathrm{C}_{c}$} & \multicolumn{1}{c}{$\mathrm{C}_{m}$} & \multicolumn{1}{c}{$\mathrm{N}$} & \multicolumn{1}{c}{$\mathrm{O}_{c}$} & \multicolumn{1}{c}{$\mathrm{O}_{t}$} \\
\hline $\mathrm{HN}$ & $-0.5 \pm 0.5$ & $0.4 \pm 0.1$ & $-2.3 \pm 0.9$ & $-1.7 \pm 1.8$ & $1.0 \pm 2.4$ \\
$\mathrm{AA}$ & $1.2 \pm 0.3$ & $-4.3 \pm 0.3$ & $-2.9 \pm 1.3$ & $-4.7 \pm 2.1$ & $-31.9 \pm 2.2$ \\
$\mathrm{OC}$ & $0.7 \pm 0.8$ & $-0.1 \pm 0.2$ & $-0.2 \pm 0.7$ & $-1.5 \pm 1.2$ & $-3.8 \pm 2.9$ \\
$\mathrm{OT}$ & $-1.3 \pm 0.6$ & $-0.1 \pm 0.3$ & $0.2 \pm 0.6$ & $-2.4 \pm 1.8$ & $3.6 \pm 3.1$ \\
\hline
\end{tabular}

For the N- conformer, the largest change corresponds to the addition of $\mathrm{H}_{2} \mathrm{O}$ at the AA site. Its effect is to deshield all nuclei (mainly $\mathrm{O}_{t}$ ), with the exception of a small increase in the shielding of $\mathrm{C}_{c}$. It is noteworthy that the values obtained for $\Delta \sigma(I, \mathrm{AA})$ are largely independent from the starting Gly. $\left(\mathrm{H}_{2} \mathrm{O}\right)_{n}$ complex. In effect, the standard deviation of the results is $28 \%, 5 \%, 23 \%, 35 \%$ and $7 \%$ of the values of $\Delta \sigma$ for the $\mathrm{C}_{c}, \mathrm{C}_{m}, \mathrm{~N}, \mathrm{O}_{c}$, $\mathrm{O}_{t}$ nuclei, respectively. Another indication of the relative irrelevance of preexistent hydrogen-bonded solvent molecules to the changes caused by the addition 
Table 5 The change in magnetic shielding (in ppm) for the non-hydrogen nuclei in Z-glycine due to the addition of a $\mathrm{H}_{2} \mathrm{O}$ molecule at the solute hydrophilic sites (see description in the text)

\begin{tabular}{crrrrr}
\hline Site & \multicolumn{1}{c}{$\mathrm{C}_{c}$} & \multicolumn{1}{c}{$\mathrm{C}_{m}$} & \multicolumn{1}{c}{$\mathrm{N}$} & \multicolumn{1}{c}{$\mathrm{O}_{c}$} & \multicolumn{1}{c}{$\mathrm{O}_{t}$} \\
\hline $\mathrm{HN}$ & $-0.8 \pm 0.3$ & $0.8 \pm 0.5$ & $-1.8 \pm 1.0$ & $0.7 \pm 3.0$ & $-1.1 \pm 2.3$ \\
$\mathrm{AA}$ & $-1.1 \pm 0.5$ & $-0.5 \pm 0.7$ & $-0.7 \pm 1.1$ & $-0.5 \pm 2.4$ & $-9.3 \pm 3.7$ \\
$\mathrm{OC}$ & $-0.5 \pm 0.6$ & $-0.1 \pm 0.8$ & $0.1 \pm 1.2$ & $2.3 \pm 3.7$ & $-6.9 \pm 4.7$ \\
$\mathrm{OT}$ & $-0.4 \pm 0.5$ & $-0.2 \pm 0.7$ & $0.1 \pm 1.0$ & $-5.0 \pm 4.5$ & $0.9 \pm 2.8$ \\
\hline
\end{tabular}

of a water molecule, is that the fluctuations in $\Delta \sigma$ are always smaller than the average $\langle\Delta \sigma\rangle$, with the exception of the few cases where $\langle\Delta \sigma\rangle$ itself is very small, namely $(\mathrm{N}, \mathrm{OC}),(\mathrm{N}, \mathrm{OT}),\left(\mathrm{C}_{m}, \mathrm{OC}\right),\left(\mathrm{C}_{m}, \mathrm{OT}\right)$ and $\left(\mathrm{C}_{c}, \mathrm{OC}\right)$.

Addition of solvent at the $\mathrm{HN}$ site mainly affects the nitrogen environment with, as expected, a deshielding similar to the AA case. In what regards the oxygen nuclei, $\mathrm{O}_{t}$ is deshielded (shielded) by the addition of $\mathrm{H}_{2} \mathrm{O}$ at the OC (OT) site, while the $\mathrm{O}_{c}$ nucleus suffers a deshielding in both cases, but smaller in absolute value.

For the Z- conformer, the changes observed when replacing the continuous description of the medium by the explicit quantum treatment of solvent molecules are smaller than for the N-conformer. The most conspicuous cases are the magnetic deshieldings for the $\left(\mathrm{AA}, \mathrm{O}_{t}\right),\left(\mathrm{OC}, \mathrm{O}_{t}\right)$ and $\left(\mathrm{OT}, \mathrm{O}_{c}\right)$ combinations, which we discuss next. Besides these, marginal changes are predicted for nitrogen and cis-oxygen when a $\mathrm{H}_{2} \mathrm{O}$ molecule is included at the $\mathrm{HN}$ and OC sites, respectively.

The carboxylate ion in the zwitterionic conformer is a hybrid of two resonance structures, with the $-\mathrm{C}-\mathrm{O}^{-1}$ negative charge either on the cis or trans oxygen atom, and the double $-\mathrm{C}=\mathrm{O}$ bond on the other. Any modification that decreases the net negative charge on oxygen, and/or increases the double nature of its bonding to the carboxylic carbon atom, will reduce its magnetic shielding because of both the smaller global electronic density and the fact that $\pi$-electrons have a vanishing value for the wavefunction at the oxygen (and $\mathrm{C}_{c}$ ) atom. This is in fact what we observe for $\mathrm{O}_{t}$ when adding explicit water molecules at the $\mathrm{AA}$ and $\mathrm{OC}$ sites, because the hydrogen-bonded water molecule stabilizes the negative charge at the OC site. The same mechanism explains the deshielding of $\mathrm{O}_{c}$ by $\mathrm{H}_{2} \mathrm{O}$ at the OT site.

\section{Concluding Remarks}

In this work we have systematically studied the nuclear magnetic shieldings of the neutral and zwitterion conformers of glycine in water solution using a dielectric continuum model of water via the PCM model and an increasingly larger number of explicit water molecules included in the quantum mechanical treatment, where all geometrical parameters are allowed to relax. 
We observed the following patterns:

- The largest effect to the magnetic shielding, in both conformers, is observed for the oxygen nuclei. The trans oxygen, $\mathrm{O}_{t}$ is the most sensitive to the presence of explicit water molecules, and the one where disagreement with the bare PCM prediction is the largest.

- For the N-conformer the $\mathrm{O}_{t}$ nucleus is systematically shielded (deshielded) when adding solvent molecules at position OT (OC), with an average change of $+6 \mathrm{ppm}(-6 \mathrm{ppm})$. The positive value is the expected behavior for an electronegative atom when hydrogen-bonded to hydrogen. Addition of $\mathrm{H}_{2} \mathrm{O}$ to the AA position brings about a large unshielding effect $(\Delta \sigma=-29.2 \mathrm{ppm})$, a result that we find surprising.

- For the Z-conformer, the two main effects are a decrease in shielding by -19 ppm and -10 ppm for the trans and the cis-oxygen, respectively. The total result for $\mathrm{O}_{t}$ arises from three negative contributions, AA $(\Delta \sigma \simeq$ $-10 \mathrm{ppm}), \mathrm{OC}(\Delta \sigma \simeq-5 \mathrm{ppm})$ and $\mathrm{HN}(\Delta \sigma \simeq-1 \mathrm{ppm})$, and one positive, $\mathrm{OT}(\Delta \sigma \simeq+2.5 \mathrm{ppm})$. The global result for $\mathrm{O}_{c}$ is smaller because of comparable positive and negative contributions, OT $(\Delta \sigma \simeq-6.5 \mathrm{ppm})$ and $\mathrm{HN}(\Delta \sigma \simeq+4 \mathrm{ppm})$.

- The shieldings tend to converge with increasing number of explicitly considered solvent molecules and for the oxygen nuclei and to a lesser extent also for the nitrogen nucleus the results differ substantially from the PCM only result. The continuum polarizable medium approximation is thus not adequate to predict NMR shieldings of a solution of glycine in water. The solvent effects are so important that the main conclusions most likely apply to other molecular properties of glycine in water, for instance to optical properties.

\section{Acknowledgements}

MCC acknowledge financial support to the present research from CONICET (PIP0369) and Universidad de Buenos Aires (UBACYT,W197), PFP acknowledge financial support from CONICET and UNNE (PI:F002-11 Res.852/11); and SPAS thanks the Danish Center for Scientific Computing (DCSC) and the TWAS Visiting Expert Programme (F.R. 3240301376) for financial support.

\section{References}

1. J. H. Jensen and M. S. Gordon. On the number of water molecules necessary to stabilize the glycine zwitterion. J. Am. Chem. Soc., 117:8159-8170, 1995.

2. A. Fernandez-Ramos, Z. Smedarchina, W. Siebrand, and M. Z. Zgierski. A directdynamics study of the zwitterion-to-neutral interconversion of glycine in aqueous solution. J. Chem. Phys., 113:9714-9721, 2000.

3. R. Karmacharya, D. Antoniou, and S. D. Schwartz. Nonequilibrium solvation and the quantum kramers problem: Proton transfer inaqueous glycine. J. Phys. Chem. A, 105:2563-2567, 2001 
4. C. M. Aikens and M. S. Gordon. Incremental solvation of nonionized and zwitterionic glycine. J. Am. Chem. Soc., 128:12835-12850, 2006.

5. K. Leung and S. B. Rempe. Ab initio molecular dynamics study of glycine intramolecular proton transfer in water. J. Chem. Phys., 122:184506(1-13), 2005.

6. M. G. Campo. Molecular dynamics simulation of glycine zwitterion in aqueous solution. J. Chem. Phys., 125:114511(1-9), 2006.

7. S. P. A. Sauer, J. Oddershede, and John R. Sabin. Directional dependence of the mean excitation energy and spectral moments of the dipole oscillator strength distribution of glycine and its zwitterion. J. Phys. Chem. A, 110:8811-8817, 2006.

8. R. M. Balabin. The first step in glycine solvation: The glycine-water complex. J. Phys. Chem. B, 114:15075-15078, 2010.

9. K. Aidas, J. Kongsted, J. R. Sabin, J. Oddershede, K. V. Mikkelsen, and S. P. A. Sauer. The Effect of Solvation on the Mean Excitation Energy of Glycine. J. Phys. Chem. Lett., 1:242-245, 2010.

10. S. Bruun-Ghalbia, S. P. A. Sauer, J. Oddershede, and J. R. Sabin. Comparison of the Directional Characteristics of Swift Ion Excitation for Two Small Biomolecules: Glycine and Alanine. Eur. Phys. J. D, 60:71-76, 2010.

11. N. Takenaka, Y. Kitamura, Y. Koyano, T. Asada, and M. Nagaoka. Reaction path optimization and vibrational frequency analysis via ab initio $\mathrm{qm} / \mathrm{mm}$ free energy gradient (feg) method: application to isomerization process of glycine in aqueous solution. Theor. Chem. Acc, 130:215-226, 2011.

12. S. P. A. Sauer, J. Oddershede, and J. R. Sabin. Mean Excitation Energies for Biomolecules: Glycine to DNA. Adv. Quantum Chem., 62:215-242, 2011.

13. J. R. Sabin, J. Oddershede, and S. P. A. Sauer. Glycine: Theory of the Interaction with Fast Ion Radiation. In W. Vojak, editor, Glycine: Biosynthesis, Physiological Functions and Commercial Uses, chapter 4, pages 79-96. Nova Science Publisher, , 2013.

14. J.-Y. Kim, D.-S. Ahn, S.-W. Park, and S. Lee. Gas phase hydration of amino acids and dipeptides: effects on the relative stability of zwitterion vs. canonical conformers. $R S C$ Adv., 4:16352-16361, 2014.

15. R. Wu and T. B. McMahon. Stabilization of Zwitterionic Structures of Amino Acids (Gly, Ala, Val, Leu, Ile, Ser and Pro) by Ammonium Ions in the Gas Phase. J. Am. Chem. Soc., 130: 3065-3078, 2008.

16. T. Hwang, G. Eom, M. Choi, S. Jang, J. Kim, and S. Le. Microsolvation of Lysine by Water: Computational Study of Stabilized Zwitterion. J. Phys. Chem. B, 115: $1014710153,2011$.

17. G. Wada, E. Tamura, M. Okina, and M. Nacamura. On the ratio of zuitterion form to uncharged form of glycine at equilibrium in various aqueous media. Bull. Chem. Soc. Jpn., 55:3064-3067, 1982.

18. M. A. Slifkin and S. M. All. Thermodinamic parameters of the activation of glycine zwitterion protonation reactions. J. Mol. Liq., 28:215-221, 1984.

19. L. A. Peteanu and D. H. Levy. Spectroscopy of complexes of tryptamine and 3indolepropionic acid with various solvents. J . Phys. Chem., 92:6554-6561, 1988.

20. S. Xu, J. M. Nilles, and K. H. Bowen. Zwitterion formation in hydrated amino acid, dipole bound anions: How many water molecules are required? J. Chem. Phys., 119:10696(1-7), 2003.

21. E. G. Diken, N. I. Hammer, and M. A. Johnson. Preparation and photoelectron spectrum of the glycine molecular anion: Assignment to a dipole-bound electron species with a high-dipole moment, non-zwitterionic form of the neutral core. J. Chem. Phys., 120:9899-9902, 2004.

22. S. Nonose, S. Iwaoka, K. Mori, Y. Shibata, and K. Fuke. Structures and reactions of hydrated biomolecular cluster ions. Eur. Phys. J. D, 34:315-319, 2005.

23. J. L. Alonso, E. J. Cocinero, A. Lesarri, M. E. Sanz, and J. C. López. The glycine-water complex. Angew. Chem., 118:3551-3554, 2006.

24. A. G. Császár. Conformers of gaseous glycine. J. Am. Chem. Soc., 114:9568-9575, 1992.

25. P. D. Godfrey, R. D. Brown, and F. M. Rodgers. The missing conformers of glycine and alanine: relaxation in seeded supersonic jets'. J. Mol. Struct., 376:65-81, 1996.

26. C. M. Aikens and M. S. Gordon. Incremental solvation of nonionized and zwitterionic glycine. J. Am. Chem. Soc., 128:12835-12850, 2006. 
27. S. Miertus, E. Scroco, and J. Tomasi. Electrostatic interaction of a solute with a continuum. a direct utilizaion of ab initio molecular potentials for the prevision of solvent effects. Chem. Phys., 55:117-129, 1981.

28. S. Miertus and J. Tomasi. Approximate evaluations of the electrostatic free energy and internal energy changes in solution processes. Chem. Phys., 65:239-245, 1982.

29. M. Cossi, V. Barone, R. Cammi, and J. Tomasi. Ab initio study of solvated molecules: A new implementation of the polarizable continuum model. Chem. Phys, Lett., 255:327335, 1996.

30. M. Cossi, V. Barone, and M. A. Robb. A direct procedure for the evaluation of solvent effects in mc-scf calculations. J. Chem. Phys., 111:5295-5302, 1999.

31. M. Cossi and V. Barone. Solvent effect on vertical electronic transitions by the polarizable continuum model. J. Chem. Phys., 112:2427-2435, 2000.

32. M. Cossi, N. Rega, G. Scalmani, and V. Barone. Polarizable dielectric model of solvation with inclusion of charge penetration effects. J. Chem. Phys., 114:5691-5701, 2001.

33. M. Cossi and V. Barone. Time-dependent density functional theory for molecules in liquid solutions. J. Chem. Phys., 115:4708-4717, 2001.

34. M. Cossi, G. Scalmani, N. Rega, and V. J. Barone. New developments in the polarizable continuum model for quantum mechanical and classical calculations on molecules in solution. J. Chem. Phys., 117:43-54, 2002.

35. M. J. Frisch, G. W. Trucks, H. B. Schlegel, G. E. Scuseria, M. A. Robb, J. R. Cheeseman, G. Scalmani, V. Barone, B. Mennucci, G. A. Petersson, H. Nakatsuji, M. Caricato, X. Li, H. P. Hratchian, A. F. Izmaylov, J. Bloino, G. Zheng, J. L. Sonnenberg, M. Hada, M. Ehara, K. Toyota, R. Fukuda, J. Hasegawa, M. Ishida, T. Nakajima, Y. Honda, O. Kitao, H. Nakai, T. Vreven, J. A. Montgomery Jr., J. E. Peralta, F. Ogliaro, M. Bearpark, J. J. Heyd, E. Brothers, K. N. Kudin, V. N. Staroverov, R. Kobayashi, J. Normand, K. Raghavachari, A. Rendell, J. C. Burant, S. S. Iyengar, J. Tomasi, M. Cossi, N. Rega, J. M. Millam, M. Klene, J. E. Knox, J. B. Cross, V. Bakken, C. Adamo, J. Jaramillo, R. Gomperts, R. E. Stratmann, O. Yazyev, A. J. Austin, R. Cammi, C. Pomelli, J. W. Ochterski, R. L. Martin, K. Morokuma, V. G. Zakrzewski, G. A. Voth, P. Salvador, J. J. Dannenberg, S. Dapprich, A. D. Daniels, Ö. Farkas, J. B. Foresman, J. V. Ortiz, J. Cioslowski, and D. J. Fox. Gaussian 09 Revision D.01. Gaussian Inc. Wallingford CT, 2009.

36. A. D. Becke. Density-functional thermochemistry. iii. the role of exact exchange. J. Chem. Phys., 98:5648-5652, 1993.

37. C. Lee, W. Yang, and R. G. Parr. Development of the colle-salvetti correlation-energy formula into a functional of the electron density. Phys. Rev. B, 37:785-789, 1988.

38. B. Mennucci and J. Tomasi. Continuum solvation models: a new approach to the problem of solute's charge distribution and cavity boundaries. J. Chem. Phys., 106:51515158, 1997.

39. W. J. Hehre, R. Ditchfield, and J. A. Pople. Self-consistent molecular orbital methods. XII. Further extensions of Gaussian-type basis sets for use in molecular orbital studies of organic molecules. J. Chem. Phys., 56:2257-2261, 1972.

40. P. C. Hariharan and J. A. Pople. Influence of polarization functions on molecular-orbital hydrogenation energies. Theor. Chem. Acc., 28:213-222, 1973.

41. F. Jensen. Segmented Contracted Basis Sets Optimized for Nuclear Magnetic Shielding. J. Chem. Theory Comp., 11:132-138, 2015.

42. N. F. Ramsey. Magnetic shielding of nuclei in molecule. Phys. Rev., 78:699-703, 1950.

43. T. Helgaker, M. Jaszuński, and K. Ruud. Ab initio methods for the calculation of nmr shielding and indirect spin-spin coupling constants. Chem. Rev., 99:293-352, 1999.

44. J. Vaara. Theory and computation of nuclear magnetic resonance parameters. Phys. Chem. Chem. Phys., 9:5399-5418, 2007.

45. S. P. A. Sauer. Molecular Electromagnetism: A Computational Chemistry Approach. Oxford University Press, Oxford, 2011.

46. T. Helgaker, S. Coriani, P. Jørgensen, K. Kristensen, J. Olsen, and K. Ruud. Recent advances in wave function-based methods of molecular-property calculations. Chem. Rev., 112:543-631, 2012.

47. K. Wolinski, J. F. Hinton, and P. Pulay. Efficient implementation of the gaugeindependent atomic orbital method for nmr chemical shift calculations. J. Am. Chem. Soc., 112:8251-8260, 1990. 
48. A. Ligabue, S. P. A. Sauer, and P. Lazzeretti. Correlated and Gauge Invariant Calculations of Nuclear Magnetic Shielding Constants Using the Continuous Transformation of the Origin of the Current Density Approach. J. Chem. Phys., 118:6830-6845, 2003.

49. A. Ligabue, S. P. A. Sauer, and P. Lazzeretti. Gauge invariant calculations of nuclear magnetic shielding constants using the continuous transformation of the origin of the current density approach. II. Density functional and coupled cluster theory. J. Chem. Phys., 126:154111, 2007. 